\title{
TTR
}

Traduction, terminologie, re?daction

\section{Définition et potentiel de dénomination d'une nomenclature}

\section{Christine Portelance}

Volume 3, numéro 1, 1er semestre 1990

L’agora de la traduction

URI : https://id.erudit.org/iderudit/037061ar

DOI : https://doi.org/10.7202/037061ar

Aller au sommaire du numéro

Éditeur(s)

Association canadienne de traductologie

ISSN

0835-8443 (imprimé)

1708-2188 (numérique)

Découvrir la revue

Citer cet article

Portelance, C. (1990). Définition et potentiel de dénomination d'une nomenclature. TTR, 3(1), 99-124. https://doi.org/10.7202/037061ar d'utilisation que vous pouvez consulter en ligne.

https://apropos.erudit.org/fr/usagers/politique-dutilisation/ 


\title{
Définition et potentiel de dénomination d'une nomenclature
}

\author{
Christine Portelance
}

Il n'y a pour une représentation synthétique et ses objets que deux manières possibles de coincider, de s'accorder d'une façon nécessaire et, pour ainsi dire, de se rencontrer. Ou bien c'est l'objet qui rend possible la représentation, ou bien c'est la représentation qui rend l'objet possible.

Emmanuel Kant, Critique de la raison pure.

La définition, problème épineux mais incontournable lorsqu'il s'agit de terminologie. Éternelle question des mots et des choses. La contribution de la syntagmatique à la construction d'un système de définitions d'un dictionnaire informatisé constitue l'objet central de cette étude.

Limprécision de la frontière entre langue naturelle (système et discours) et organisation conceptuelle rend le travail de réflexion ardu, mais mérite cependant qu'on s'y attarde. C'est pourquoi nous traiterons tout d'abord des questions théoriques sous-jacentes à la définition, en tenant compte des théories sémantiques, de la philosophie du langage et de la philosophie des sciences, sans toutefois prétendre à l'exhaustivité sur un sujet aussi vaste. Cette section a pour but de dégager les caractéristiques propres à une définition «terminologique». 
En deuxième partie, à partir de certains résultats d'une étude syntagmatique portant sur les mécanismes linguistiques de la dénomination, nous proposons une nouvelle structure de définition mettant au jour la dynamique interne d'une nomenclature, c'est-àdire son potentiel de dénomination. Les données servant à illustrer l'existence de ce potentiel ont déjà été présentées dans un colloque de linguistique quantitative (Portelance, 1989), mais elles n'ont jamais auparavant été mises en relation avec les problèmes théoriques de la définition.

Pour ne pas alourdir indûment la présente étude, nous ne ferons pas une présentation complète de nos travaux en syntagmatique. Seuls les éléments en rapport avec les problèmes liés à la définition seront ici exposés. Nous réservons pour publication ultérieure un panorama critique des différents travaux en la matière (notamment Guilbert, 1965, 1967, 1975; Auger, 1978; Dubuc, 1978; Boulanger, 1988), ainsi que la question globale d'une théorie de la syntagmatique applicable aux langues de spécialité.

\section{Définition et problèmes théoriques}

\section{Définition et description}

Pour A. Rey (1979), le problème de la définition peut constituer le centre des questions terminologiques et il le pose de deux manières: la définition comme opération logique et comme opération langagière, et la différence entre définition et description.

Rey oppose la définition ontologique d'Aristote à la conception de la définition de Kant, pour qui seule la mathématique possède des définitions, puisqu'elle seule construit des concepts à partir d'une synthèse arbitraire. Quoi qu'il en soit, Rey réunit les deux points de vue:

De la définition ontologique d'Atistote à la définition constructive (fonctionnelle) de la mathématique et des systèmes formels, on reste sur le même terrain: on définit non pas des mots mais des termes organisés en systèmes structurés et reflétant une organisation conceptuelle formelle (consistante), qu'elle soit considérée ou non comme reflétant les structures mêmes de l'être. (p. 41)

Rey établit la distinction entre définition et description en affirmant que la première doit fournir tous les traits pertinents de la signification (définition linguistique) ou les traits conceptuels pertinents et seulement eux, alors que la deuxième peut cumuler traits pertinents et traits caractéristiques. Il note que bien des définitions de dictionnaires encyclopédiques et même linguistiques ne sont en fait que des descriptions. 
Si Rey (1979) donne comme définition d'une terminologie l'association d'un système de termes et d'un système définitionnel, il ne fournit cependant pas de description d'une telle association. Il affirme qu'empiriquement une définition terminologique est un compromis entre une définition lexicographique et une définition encyclopédique et qu'une telle définition se construit en compréhension plutôt qu'en extension, ce qui est, somme toute, bien peu sur le plan pratique. Il conclut qu'une définition terminologique pure, dans tous les domaines, est impraticable et en cela, il est conséquent avec lui-même puisqu'il consacre tout un chapitre dans son ouvrage le Lexique: images et modèles à «l'impossible définition» (entendre définition lexicographique). Si la définition terminologique comporte des traits descriptifs, on peut penser que la nature, le nombre et l'organisation de ces traits peuvent varier en fonction du domaine traité.

\section{Scientifique? Technique? Technoscientifique?}

Selon Rey, la terminologie telle qu'elle se pratique actuellement ne peut s'appliquer aux sciences pures, elle reste donc limitée aux sciences appliquées et à la technologie. Sur ce dernier point, nous ne pouvons être d'accord car il est difficile d'opérer une division nette entre science et technique. En effet, l'étude diachronique de terminologies montre bien l'importance de l'interaction entre différentes disciplines lors de l'émergence d'une technique entièrement nouvelle. Comme Guilbert (1965) a pu l'observer, le lexique de l'aviation entretient des rapports avec le lexique de la physique, de la géométrie, de l'ornithologie, de l'entomologie, de l'anatomie, du transport maritime et du transport ferroviaire. Vouloir cantonner la terminologie à la description de termes techniques constitue une erreur d'orientation de la discipline, puisque c'est risquer de la condamner ainsi à graviter autour d'activités langagières prescriptives qui ont peu à voir avec la transmission et la circulation du savoir.

La nomenclature utilisée comme corpus d'analyse est du domaine de l'électrotechnique: certaines notions viennent de la physique, d'autres relèvent des applications techniques. Pour ces raisons, nous pensons que la terminographie doit également se préoccuper des questions scientifiques et que, pour garantir une rigueur méthodologique, les systèmes définitionnels produits par la terminographie doivent servir aussi bien à l'encodage qu'au décodage. En effet, idéalement, les dictionnaires technoscientifiques devraient être conçus en premier lieu pour les spécialistes d'un domaine pour ensuite être utilisés pour la production de lexiques et de vocabulaires utiles à la traduction et, éventuelle- 
ment, afin de servir à la génération de textes et à l'indexation automatique. Dans bien des cas, on trouve dans les dictionnaires techniques actuels des définitions terminologiques dont la forme ne sert souvent qu'à établir l'équivalence entre des termes de langues différentes.

Rey propose que la définition terminologique soit un compromis entre la définition linguistique et la définition encyclopédique, mais encore faut-il être en mesure de les distinguer. Question litigieuse et propice à la controverse, si l'on pense seulement à la forme que peut prendre une définition linguistique dans différents travaux en sémantique: Greimas (1966), Jackendoff (1983), Wierzbicka (1985), Mel'ćuk (1984; 1988), etc.

\section{Dictionnaire vs encyclopédie}

Pour aborder la question de la définition terminologique sans la minimiser, il faut reprendre la discussion de la différence entre dictionnaire et encyclopédie. Haiman (1980) s'est intéressé à cette question; il attaque le sujet en passant en revue les distinctions généralement utilisées pour opposer les concepts de dictionnaire et d'encyclopédie, distinctions qu'il réfute les unes après les autres. Il qualifie les concepts d' '(essentiel» et d' «accidentel» d'Aristote et les concepts de "vérité analytique» et de "vérité synthétique» chez Kant d'arbitraires, donc d'inutilisables; il considère que la différence entre le savoir linguistique et le savoir culturel ou encore l'opposition nom commun/nom propre est non pertinente.

Semantic knowledge derives from cultural knowledge; it is a subset of knowledges, with rather fuzzy boundaries. (p. 335)

Pour Haiman, il n'y a de pas de différence à faire entre dictionnaire et en'cyclopédie; sur le plan théorique, les dictionnaires sont des encyclopédies. Il admet toutefois que, sur le plan pratique, il y aura toujours une mise en marché des deux types d'ouvrages. Une fois les notions servant à opposer dictionnaire et encyclopédie rejetées, il aborde une question de linguistique théorique intéressante, dernier argument servant à justifier sa position. Il rappelle qu'à une certaine époque du structuralisme américain la division de la grammaire en phonologie, morphologie et syntaxe, établie pour des raisons pratiques, a été élevée au statut de principe théorique jusqu'à ce que Chomsky démontre que la «separation of levels» n'est pas un principe valable. Haiman estime que de nos jours le même piège guette la division de la sémiotique en syntaxe, sémantique et pragmatique dont une des conséquences visibles serait justement la séparation entre dictionnaire et encyclopédie. 


\section{Définition terminologique et linguistique}

La question n'est pas facile à traiter, étant donné l'absence de consensus chez les linguistes que la sémantique intéresse. Comparons, par exemple, deux affirmations concernant la dénotation:

Relations of sense in themselves are useless unless the words are at some point anchored in reality. Denotation is the basis of meaning. (Haiman, 1980, p. 336)

(...) lexicography is concerned not with 'denotational structure' but with conceptual structure, not with 'denotational conditions' but with meaning. (Wierzbicka, 1985, p. 15)

Il faut en premier lieu tenir compte du contexte de ces deux citations, elles sont tirées d'études poursuivant des buts différents. Haiman traite de l'opposition dictionnaire et encyclopédie sur un plan strictement théorique, sans donner d'exemples concrets de définitions, et sans même décrire ce que devrait être une définition, alors que Wierzbicka s'intéresse non seulement au concept de définition, mais elle fournit également des exemples de définitions de dictionnaires bâties à partir de ce qu'elle nomme l'analyse conceptuelle.

A l'examen des travaux de cette dernière, il apparaît que ces deux auteurs ne sont pas en pleine contradiction et nous entendons montrer où ils peuvent se rejoindre en procédant à une présentation $^{1}$ du travail de Wierzbicka à partir d'une sélection de ses exemples.

Prenons pour commencer l'exemple que Wierzbicka donne du mot plant. Pour l'ensemble des locuteurs de l'anglais, ce mot désigne ce qui pousse hors de terre et qui est de petite taille, ce qui exclut les arbres. Pour un botaniste, la catégorie "plante» comprend les arbres. Wierzbicka affirme que les locuteurs connaissent la différence entre plant de la langue générale et plant de la langue scientifique. Si les locuteurs ('people') emploient différemment ce mot, ce n'est pas par ignorance, mais parce qu'ils utilisent des critères de classification différents (p. 154); en fait, ils connaissent généralement les deux concepts. Défini selon certains modèles sémantiques, ce mot serait analysé en deux lexèmes et apparaîtrait donc deux fois dans le lexique.

1. Présentation simplifiée pour les besoins de l'exposé. 
Pour introduire la notion de «concept maximum», Wierzbicka présente son concept personnel de «potatoe». Selon l'expérience qu'elle a des pommes de terre, elle en connaît le goût et l'aspect, elle sait qu'on les récolte sous terre, qu'elles poussent en groupe et que la plante peut présenter une floraison. Par contre, elle dit connaître moins de choses sur les courgettes; elle en connaît bien l'aspect et le goût, elle s'imagine qu'elles poussent hors de terre et non dans des arbres. Elle a donc l'impression que son concept de «pomme de terre» est à peu près complet, mais que celui de "courgette» est incomplet. La situation pourrait être inverse chez d'autres locuteurs. Elle émet donc l'hypothèse de l'existence de «concept maximum» pour de tels mots. (p. 215)

Dans ces deux exemples, la situation n'est pas la même: dans le premier cas (plant), il y a deux analyses conceptuelles, deux concepts, il faut donc y voir deux lexèmes; dans le deuxième cas, c'est la qualité de l'analyse conceptuelle qui diffère selon l'expérience que le locuteur possède du concept et cela n'a pas pour effet d'entraîner la création de lexèmes différents. Examinons un autre exemple, celui de vent ${ }^{2}$ : en météorologie, ce mot possède indéniablement deux actants, la direction et la vitesse, (un vent du nordouest de $50 \mathrm{~km} / \mathrm{h}$ ) ce qui n'est pas le cas du même mot en langue générale. La notion de "concept maximum» semble très importante pour la définition terminologique, puisqu'elle constitue, selon nous, le but à atteindre.

Dans la section Meaning and Scientific Knowledge: Looking for Evidence de son livre, Wierzbicka fait référence aux travaux d'Apresjan par le biais de longues citations. La pertinence de l'une d'entre elles pour notre discussion en justifie le résumé suivant. Selon Apresjan, la sémantique d'un signe reflète l'idée naïve que l'on se fait d'une chose, d'une propriété, d'une action, etc. Il croit qu'une image naïve du monde se forme au fil des siècles, image différente de l'image scientifique du monde, et que dans cette image naïve existe une géométrie naïve, une psychologie naïve, etc. Le sens (lexical) des mots reflète le système d'explications rattaché à cette image. Aussi Apresjan est-il convaincu que le travail du lexicographe consiste à révéler cette image dissimulée sous la couverture des mots. Il croit que le lexicographe doit écarter l'image scientifique du monde puisqu'elle est indépendante de la langue qui sert à la décrire.

2. Cet exemple nous a été foumi par Alain Polguère. 
Étant donné le volume des données en cause, nous comprenons que les lexicologues délèguent aux terminologues le soin de s'occuper de l'image scientifique (ou technoscientifique) du monde. Toutefois, cette image n'est pas entièrement indépendante de la langue et encore moins du langage, si l'on considère la mathématique comme un langage ${ }^{3}$. Le premier argument que nous voulons évoquer pour expliquer notre point de vue est celui de la non-universalité de l'opposition entre langue générale et langue scientifique. Cette dichotomie est propre aux cultures que Wierzbicka qualifie de «science oriented cultures», ce qui est le cas de la culture occidentale.

Prenons comme exemples les notions de «carburateur», de «mémoire» (d'ordinateur) et de «relativité». Une grande partie des automobilistes peuvent dire: « j'ai fait ajuster mon carburateur», tout en ayant un concept tout à fait minimal du carburateur, du type: partie du moteur, a quelque chose à avoir avec l'essence. De même, l'ordinateur étant un outil répandu dans le domaine du travail de bureau, certains utilisateurs sont probablement incapables de distinguer la mémoire vive de l'ordinateur de la mémoire secondaire de leur disque rigide interne, ce qui ne les empêche pas d'utiliser un ordinateur... et le mot mémoire. Pour de tels utilisateurs, l'ordinateur, tout comme le téléphone d'ailleurs, est une sorte de mystérieuse boîte noire dont ils connaissent l'emploi. Dans ces deux cas, il est facile d'imaginer toute une échelle de concepts possibles avant d'atteindre le concept maximum, échelle reflétant la grande variété d'expérience de ces concepts. Les concepts maxima de "carburateur» et de «mémoire» doivent posséder une définition terminologique, c'est-à-dire une définition dans leur domaine respectif, soit la mécanique automobile et l'informatique.

L'individu moyen connaît le mot relativité qu'il peut généralement relier au nom d'Einstein et il sait que ce concept concerne une théorie scientifique sans être toutefois en mesure d'en exposer la teneur. Le concept maximum de "relativité» doit être défini dans le domaine de la physique. Il existe toutefois une différence entre la définition des deux premiers concepts «mémoire» et «ordinateur» et celui de «relativité». Pour ce dernier, un plus grand nombre de notions sera nécessaire pour en cerner le contenu, et il paraît difficile d'éviter le recours à la mathématique. Néanmoins, le procédé de définition sera dans l'ensemble le même: «carburateur» doit être défini en rapport avec les autres notions de mécanique automobile, «mémoire» par rapport à celles de l'informatique et

3. Dans la citation d'Apresjan (texte anglais traduit du russe), l'utilisation du mot «language» pourrait éventuellement porter à équivoque. 
«relativité» par rapport à la physique. Ce qui diffère, ce n'est pas le procédé de définition, mais bien le type d'expérience propre à chaque domaine.

Lorsque Wierzbicka nous offre des définitions, elle tente d'exprimer l'expérience partagée par l'ensemble des locuteurs d'une langue au sujet d'un concept; c'est pourquoi elle affirme qu'une définition doit se faire par analyse conceptuelle. Son travail correspond tout à fait à la définition du travail lexicographique fournie par Apresjan.

\section{Langue générale/langue de spécialité}

Lopposition entre langue générale ${ }^{4}$ et langue de spécialité ne peut se faire d'un point de vue linguistique, elle ne peut s'établir qu'à partir de la notion d'expérience partagée par des locuteurs. L'opposition sous-jacente est plutôt: expérience partagée par l'ensemble des locuteurs d'une langue/expérience partagée par des sousensembles des locuteurs d'une langue. La différence appartient au domaine de la pragmatique et c'est à partir d'une telle constatation que nous croyons que la position de Haiman n'est pas nécessairement en contradiction avec celle de Wierzbicka.

Par ailleurs, pour utiliser une opposition entre langue générale/langue scientifique à la Apresjan, il faut se situer en synchronie parce que dans une perspective diachronique, les traits [naif] et [non naif] pour qualifier l'expérience du monde perdent de leur pertinence. Le concept «système solaire» d'avant Copernic fait partie d'une physique naïve si on le considère à partir des théories physiques actuelles. En pensant aux changements successifs apportés à la physique par Kepler, Galilée, Newton et Einstein, on peut se permettre d'affirmer qu'on est toujours naif par rapport à quelqu'un. De plus, Polanyi (1952) traite des découvertes en physique mathématique motivée par la seule «beauté» ${ }^{5}$ des équations; et, à propos de la valeur de vérité des théories scientifiques, il déclare:

To hold a natural law to be true is to believe that its presence will manifest itself in an indeterminate range of unknown and perhaps yet unthinkable consequences. It is to regard the law as a real feature of nature which, as such, exists beyond our control. (...) Real is that which is expected to reveal itself indeterminately in the future. $(1964, \mathrm{p} .10)$

\footnotetext{
4. Wierzbicka utilise tantôt ordinary English, tantôt natural English ou non scientific English.
}

5. Lauteur utilise l'expression pure theoretical beauty. 
Si l'analyse linguistique des termes d'une théorie des sciences dites «dures» est ardue, ce n'est pas à cause du lien privilégié avec le référent, puisque celui-ci peut être tout à fait hypothétique, mais plutôt parce que le métalangage servant aux définitions est mathématique.

La difficulté d'établir un langage neutre, élaboré à des fins de description scientifique, de modélisation, est bien connu des linguistes, mais ce problème se pose dans toutes les sphères d'activités scientifiques. Depuis les Grecs, ce sont les développements de la mathématique qui ont permis l'existence de ce qu'on nomme aujourd'hui les sciences dures. Pour Lichnerowicz (1987), les mathématiques ont constitué au point de départ une tentative de créer «un discours capable, par sa forme même, d'interdire le refus de son contenu", d'où l'idée de démonstration. Plusieurs cultures différentes ont contribué à l'édification des mathématiques; c'est pourquoi, pour ce mathématicien-physicien, elles sont universelles, du moins certains concepts: «Entre des ensembles convenables, il peut exister des sortes de dictionnaires parfaits ${ }^{6} "$, d'où l'élimination de paradoxes. On reconnaît là la position de Kant pour qui seules les mathématiques connaissent de véritables définitions. Du point de vue de Lichnerowicz, les langues naturelles sont inadéquates à rendre les concepts d'une théorie physique (puisqu'une théorie ne saurait avoir deux sens, un sens ésotérique et un sens vulgaire) et ne peuvent servir que de stimuli à l'imagination, ce qui n'est tout de même pas négligeable.

Toute tentative d'expression en langue vulgaire d'une théorie physique est, peu ou prou, à base d'analogies variables selon les cultures et capables de nous trahir, analogies qui ne disposent que d'un pouvoir heuristique pouvant stimuler notre imagination. (Lichnerowicz, 1987, p. 242)

Lorsqu'il parle de trahison, est-ce sous-entendre que les langues naturelles peuvent dissimuler le réel ou la réalité? Ce serait alors prétendre que les sciences pures décrivent la réalité, ce qui est contestable et ce, depuis Kant. La trahison que peuvent entraîner les mots consiste plutôt en une entrave au développement de modèles, et il ne faut pas oublier non plus qu'en physique on tient maintenant compte de l'existence de l'observateur, donc d'un certain degré de subjectivité. Pour qu'il ait science normale ou paradigme (matrice disciplinaire), concept proposé par Kuhn (1970), il faut qu'il y ait consensus autour d'une théorie. Or, la mathématique sert à élaborer des systèmes de concepts qui permettent d'établir des consensus. Lichnerowicz note toutefois que la

6. En italique dans le texte de Lichnerowicz. 
mathématique est plus qu'un langage, et qu'elle n'est donc pas neutre puisqu'elle constitue, en tant que discipline, un mode de pensée. La mathématique possède également ses limites, comme on peut le constater dans les derniers développements de la mécanique quantique, laquelle, quoique fondée sur des concepts mathématiques, est également incohérente du point de vue mathématique. Lichnerowicz attribue cet état de choses au caractère encore provisoire du modèle:

Notre approche théorique est certainement encore provisoire.

Mais cette théorie nous a fourni un tel facteur d'intelligence et de contrôle du monde microscopique qu'elle apparaît comme la théorie physique la plus fondamentale et la plus insatisfaisante. (p. 256)

Et Kuhn pourrait ajouter: jusqu'à ce que l'insatisfaction engendre un état de crise entraînant une révolution scientifique, un changement de paradigme (matrice disciplinaire).

Les limites de l'adéquation des mathématiques à fournir une modélisation cohérente des observables sont fixées par notre propre capacité à déterminer ces observables et ainsi construire un monde scientifique. C'est pourquoi nous ne sommes pas près de nous passer des mots et de leur pouvoir heuristique. Les langues naturelles, malgré leur ambiguïté intrinsèque, demeurent essentielles au développement de notre pensée scientifique, reflet d'une communauté qui ne pourrait exister sans la communication verbale: transmission du savoir, échange de points de vue. On ne bâtit peut-être pas une théorie physique par la discussion, mais l'on n'a jamais vu de théorie naître sans l'existence d'une école ou d'une communauté de pensée.

\section{Sens et référence}

On ne peut aborder la définition sans traiter du concept de référence. Il est généralement admis, dans la plupart des théories sémantiques, que le référent d'un signe linguistique est sa correspondance avec la réalité extra-linguistique. Cette définition est insatisfaisante à cause des problèmes engendrés par la valeur de vérité des énoncés. Nous choisissons plutôt une approche comme celle de Jackendoff (1983), même si elle constitue l'élément le plus controversé de sa théorie. Son modèle sémantique comporte une contrainte grammaticale et une contrainte cognitive; la théorie de la référence participe de la deuxième.

Jackendoff, s'appuyant sur les acquis de la psychologie, rapproche la faculté de langage des systèmes de perceptions visuelle (couleur) et auditive (musique). Les productions linguistiques, comme musicales, ne peuvent exister sans créateur humain 
et correspondent à une structure mentale. Par conséquent, l'information véhiculée par le langage ne constitue pas de l'information à propos de la réalité mais plutôt de l'information concernant une projection de la réalité, ce qui l'amène à affirmer:

Perhaps the most fundamental consequence of the approach adopted here is that the standard notions of truth and reference play no significant role in natural language semantics. (p. $x$ de l'introduction)

Nous préférons, à propos de cette dernière affirmation, adopter une attitude plus nuancée, en renvoyant à Popper (1960) qui, à la suite des théories de Xénophane et de Tarski, affirme:

(...) that we search for truth, but may not know when we have found it; that we have no criterion of truth, but are nevertheless guided by the idea of truth as a regulative principle (as Kant or Peirce might have said) (...) there are something like criteria of progress towards the truth. (p. 185)

Le contre-argument généralement invoqué à propos d'une telle approche de l'information linguistique est qu'un tel degré de subjectivité de la langue empêcherait la communication; à cela, Jackendoff rétorque que les processus qui servent à bâtir cette projection de la réalité sont les mêmes pour tous et que l'obstacle dans la communication d'information entre individus vient plutôt d'interprétations d'expérience incompatibles. Et il conclut:

Thus we can reasonably operate under the assumption that we are talking about the same things, as long as we are vigilant about detecting misunderstanding. (p. 31)

Les pratiques terminologiques font justement partie de la vigilance requise pour garantir une bonne circulation de l'information. En cela, la terminologie peut constituer, pour reprendre une expression d'Alain Rey, une thérapeutique du discours.

La terminologie a pour tradition de travailler par domaine d'activité et cela constitue un point important pour conserver la plus grande rigueur. Pour un même référent, la spécialisation peut entraîner des définitions différentes. En demandant à deux éminents chercheurs, un chimiste et un physicien, si un atome d'hélium constitue une molécule, on obtint deux réponses catégoriques, mais différentes. Pour le chimiste, oui sans hésitation, puisque cet atome se comporte comme une molécule du point de vue de la théorie cinétique des gaz. Pour le physicien, non sans hésitation, puisque cet atome ne permet pas d'apercevoir un spectre moléculaire. 7

7. Ces exemples sont empruntés à Kuhn (1970). 
La difficulté de la définition terminologique tient également à son caractère non stable. Entre deux théories scientifiques successives ou concurrentes, les chercheurs utilisent souvent les même termes, mais les rapports qu'entretiennent entre eux ces termes sont différents. En fait, ce n'est qu'après l'expérience scientifique que 1 'on peut commencer à chercher une définition véritablement opérationnelle. Tant que l'expérience, avec toutes les vérifications que l'opération exige, n'est pas complétée, un terme n'est pas «dé-fini», c'est-à-dire que l'expérience n'a pas encore permis de lui assigner une limite. De plus, la technologie actuelle crée et modifie sans cesse ses produits. Il y a de quoi sourire à l'idée que les premiers micro-ordinateurs personnels «performants» n'avaient que $16 \mathrm{k}$ de mémoire vive!

Un autre point dont on doit tenir compte dans l'élaboration des définitions terminologiques technoscientifiques est le type d'apprentissage que nécessite la formation scientifique et technique. Ce point est traité par Kuhn (1969):

Ce genre de connaissance ne s'acquiert pas exclusivement par des moyens verbaux. Au contraire quelqu'un y parvient quand on lui donne des termes en même temps que des exemples concrets de leur mise en pratique; la nature et les termes s'apprennent ensemble. (p. 260)

Kuhn utilise l'expression de Polanyi «connaissance tacite» pour qualifier le résultat de ce processus; cette connaissance tacite s'acquiert en faisant de la science plutôt qu'en apprenant des règles pour en faire. C'est donc dire qu'un système définitionnel n'a pas à viser l'exhaustivité, ce qui rendrait la tâche impossible. En conséquence, contrairement aux aspirations de certains lexicologues qui aimeraient tout définir, avec les problèmes métalinguistiques des primitifs que cela entraîne, un système définitionnel terminologique présuppose une sélection de ce qui est à définir ou à décrire selon les niveaux d'organisation de la nomenclature. La définition du concept mathématique de nombre dans le Petit Robert est un bel exemple d'une impossible définition:

concept de base des mathématiques, une des notions fondamentales de l'entendement que l'on peut rapporter à d'autres idées, mais non définir.

A ce sujet, le logicien Frege a écrit:

On ne saurait demander que tout soit défini (...). Ce qui est simple ne peut pas être analysé, et ce qui est logiquement simple ne peut être véritablement défini et cela qui est logi- 
quement simple n'est pas donné d'avance (...), c'est le fruit d'une recherche scientifique. ${ }^{8}$

Les concepts d'une discipline qui ne peuvent être vraiment définis sont appréhendés par les spécialistes du domaine par la voie de la «connaissance tacite» et sont considérés par les non-spécialistes comme des a priori.

\section{Potentiel de dénomination d'une nomenclature}

\section{Système de notions et système de définitions}

Si l'on essaie de concevoir le système de définitions qui doit accompagner un système de termes, il faut considérer la structure de la nomenclature et ses niveaux d'organisation. La définition d'un terme doit tenir compte de la relation entretenue par la notion avec le système notionnel. C'est donc dire qu'une telle définition doit non seulement tenir compte de la signification du terme mais également du concept saussurien de valeur. Puisqu'un système notionnel possède une structure sous-tendue par un réseau de liens entre les notions, toutes les définitions ne doivent pas nécessairement être de construction identique. La définition d'un terme ne doit pas viser l'exhaustivité du point de vue de la signification, mais elle doit être complète du point de vue de la valeur, laquelle est déterminée par le système de termes d'un domaine. Il faut toutefois admettre que l'acte de définition des notions d'un domaine comporte des limites, contraintes occasionnées par l'existence de concepts a priori déterminés par le type d'expérience impliqué dans un champ d'investigation scientifique.

\section{Structure linguistique d'une nomenclature et dénomination}

La question de la dénomination', c'est-à-dire le mécanisme linguistique du processus de désignation, a été abordée par le biais d'une étude de syntagmatique. Pour ce faire, plus de 8000 syntagmes dans le domaine de l'électrotechnique ont été examinés en tentant de dégager les structures linguistiques de la nomenclature. Pour être en mesure d'offrir une description lexico-syntaxique de ces termes et appuyer de statistiques les généralisations proposées, nous avons procédé à un recensement de tous les modèles syntaxiques ainsi qu'à un inventaire lexical.

8. Cité par Rey (1979: 38 (1)).

9. Naming en anglais. 
Au plan syntagmatique, devant le nombre considérable de configurations, l'examen par tranche a été effectué en utilisant comme caractéristique de classement le nombre de substantifs présents dans chaque syntagme. Chaque classe de termes a été examinée à l'aide d'une matrice théorique de description établie à partir de la liste de toutes les configurations syntagmatiques et capable de reproduire toutes les matrices terminogéniques.

Les appellations métalinguistiques des classes de termes consistent en une série descriptive basée sur le nombre de substantifs: mononominaux, binominaux, trinominaux, quadrinominaux, quinominaux, sextinominaux. Ce type de classement a déjà été utilisé pour un autre corpus de 5000 termes français qui sert dans cette étude de corpus de référence (Portelance, 1987).

Exemples:

$\begin{array}{ll}\text { Mononominaux: } & \begin{array}{l}\text { émission électronique secondaire } \\ \text { Binominaux: } \\ \text { Trinominssion à double bande latérale } \\ \text { amplificateur d̀ seuil pour courant } \\ \text { continu }\end{array} \\ \text { Quadrinominaux: } & \begin{array}{l}\text { alimentation en cascade de relais de } \\ \text { voie }\end{array} \\ \text { Quinominaux: } & \begin{array}{l}\text { perte de conversion d'un chargeur de } \\ \text { fréquence d cristal } \\ \text { récepteur traducteur imprimeur pour le } \\ \text { code Morse et ses dérivés }\end{array}\end{array}$

L'étude du vocabulaire de la liste de termes a permis d'identifier le type de combinatoire en jeu dans une nomenclature et a mis en lumière l'importance de l'imbrication des plans syntagmatique et paradigmatique dans un système de termes.

\section{Le plan syntagmatique}

Il y a 8395 syntagmes, sur les 10300 de la liste, qui constituent des syntagmes de catégorie [substantif], soit $81,5 \%$ de l'ensemble de termes. Ces termes prennent la forme de 296 configurations syntagmatiques différentes, mais certaines matrices terminogéniques sont privilégiées. Onze matrices terminogéniques totalisent à elles seules $82,2 \%$ des syntagmes substantifs. Parmi celles-ci, deux matrices sont nettement dominantes: $[\mathrm{N}+$ Adj] et $[\mathrm{N}+$ Prép $+\mathrm{N}]$ et représentent $56,5 \%$ des termes.

Examinons maintenant à partir de quels ajouts on peut obtenir une matrice théorique de description englobant $82,2 \%$ des termes.

Si l'on tient compte de la possibilibité d'une détermination asyndétique, $\mathrm{N}+$ (prép) $+(\mathrm{N})$, on passe de $56,5 \%$ à $60 \%$. En ajoutant un adjectif, $\mathrm{N}+(\mathrm{Adj})+(\mathrm{PR})+(\mathrm{N})+(\mathrm{Adj})$, on passe à $73 \%$. 
Si l'on ajoute à la matrice un $\mathrm{N}$ avec ou sans préposition, $\mathrm{N}+$ (Adj) $+($ Prép) $+(\mathrm{N})+(\mathrm{AJ})+($ Prép $)+(\mathrm{N})$, on obtient $78 \%$, et c'est l'addition de déterminants $\mathrm{N}+(\mathrm{AJ})+($ Prép) $+($ Dét $)+(\mathrm{N})+($ Adj) $+($ Prép $)+($ Dét $)+(\mathrm{N})$ qui porte le total à $82,2 \%$. On obtient de cette manière la matrice théorique de description suivante:

$[\mathrm{N}+($ Adj $)+($ Prép) $+($ Dét $)+(\mathrm{N})+($ Adj $)+($ Prép) $+($ Dét $)+(\mathrm{N})]$

Le terme le plus long de notre liste comporte six éléments (en omettant prépositions, conjonctions et autres mots vides) et il fait partie de la classe des sextinominaux. Or, le terme le plus long de la classe des mononominaux est formé de cinq éléments. La longueur limite des termes de notre corpus est donc de cinq déterminations. Plus il y a de déterminations [Prép $+\mathrm{N}]$ dans un syntagme terminologique, moins il y a d'adjectifs, malgré la possibilité théorique de déterminer chaque [N] par un adjectif et chaque [Adj] par un adverbe.

Il y a 153 matrices qui ne produisent qu'un seul terme et 56 qui en produisent deux. D'un point de vue statistique, on peut penser que de telles données sont négligeables et que toutes ces configurations ne sont que bizarreries et exceptions. Or, nous avons constaté le même phénomène dans d'autres vocabulaires de taille et de facture différentes (Portelance, 1987), il faut donc chercher une explication en examinant les termes sous un autre angle, c'est-à-dire en considérant les termes dans leurs relations paradigmatiques (Portelance, 1989).

\section{Les relations paradigmatiques}

La saturation du vocabulaire, c'est-à-dire la relation entre l'étendue d'un texte $\mathrm{N}$ (nombre de mots dont le texte est constitué) et l'étendue du vocabulaire $V$ (nombre de vocables dans le texte) a été étudiée par plusieurs chercheurs, notamment par Charles Muller (1977). On observe que V croît avec $N$, mais que cet accroissement diminue avec l'augmentation de $\mathrm{N}$. Un texte continu français de 15000 mots contient environ $10 \%$ de vocables. Un phénomène similaire est observable avec une liste de termes tirée d'un lexique spécialisé, si l'on considère cette liste comme du matériel textuel et les mots dont les termes sont formés comme le vocabulaire. En examinant par tranche la liste des 10300 termes d'où sont tirés les syntagmes, l'accroissement du vocabulaire est représenté par les données du tableau ci-dessous. Les statistiques 
ont été obtenues dans le cadre du projet $\mathrm{BATEM}^{10}$ et diffusées par Baudot (1988).

\begin{tabular}{lrrrrrr}
\hline $\begin{array}{l}\text { Nombre cumulatif de } \\
\text { termes }\end{array}$ & 500 & 995 & 2000 & 3990 & 7976 & 10300 \\
\hline Nombre de mots N: & 1305 & 2647 & 5439 & 11462 & 23238 & 30479 \\
Nombre de mots diff. V: & 486 & 896 & 1566 & 2532 & 3949 & 4632 \\
V/N et \%: & 37,2 & 33,8 & 28,8 & 22,2 & 17,0 & 15,2 \\
\hline
\end{tabular}

Le nombre de mots graphiques croît avec le nombre de termes, mais cet accroissement est de plus en faible au fur et à mesure que les termes augmentent: le pourcentage de mots différents est de 33,8\% pour 1000 termes et il tombe à $15 \%$ pour 10300 termes. Or, on s'attendrait à ce que le vocabulaire au sens graphique soit plus élevé. Ce taux de saturation est comparable à celui des textes continus et Baudot (1988) attribue cet état de fait à trois facteurs: $1^{\circ}$ certaines catégories de mots sont privilégiées, $2^{\circ}$ les termes sont puisés à même un réservoir limité, $3^{\circ}$ les syntagmes terminologiques ont une formation syntaxique plus figée.

En effet, 83\% des termes de cette liste forment des syntagmes dont les constituants se combinent selon certains modèles syntaxiques, et des constituants identiques se retrouvent dans de nombreux termes. L'examen des matrices terminogéniques a permis de voir en quoi consiste la formation syntaxique plus figée et les catégories de mots dont les termes sont constitués. Examinons maintenant quelle est la nature du «réservoir limité de mots».

\section{Inventaire lexical}

Les 10300 termes sont formés de 30479 mots dont 4632 formes différentes (graphies différentes), ce qui veut dire deux fois plus ${ }^{11}$ de termes que de mots différents. Il y a donc répétition de certains mots, comme l'illustre le tableau ci-dessous.

En soustrayant les mots vides du vocabulaire, on obtient 22 833 occurrences et $\mathbf{4 6 0 8}$ formes différentes, et le taux de saturation grimpe de $15,2 \%$ à $20,2 \%$. Voyons de quelle manière se répètent les mots différents.

Une fois les mots vides retirés, les 100 mots les plus fréquents ( $0,2 \%$ des mots différents) correspondent à 6820 occurrences, soit $30 \%$ des occurrences. On y retrouve huit adjectifs: électrique,

10. BATEM est un logiciel de minibanque de données terminologiques fonctionnant sur micro-ordinateur. Ce logiciel a été mis au point à l'Université de Montréal.

11. Plus précisément 2,23 fois plus. 
magnétique, automatique, électronique, acoustique, continu, inverse et radioélectrique. Il y a également non qui peut être soit adverbe, soit nom comme dans circuit NON. Sur les 91 substantifs restants, 57 sont à la fois termes et constituants de termes; ce sont, par ordre de fréquence:

courant (304); tension (255); circuit (185); puissance (162); contact (136); signal (121); duree (100); charge (96); commande (95); électrode (95) relais (95); élément (93); rayonnement (90); résistance (89); enroulement (88); réseau (79); antenne (76); action (69); réponse (68); champ (67); énergie (65); protection (64); compteur (62); dose (61); convertisseur (60); bruit (59); enregistrement (59); erreur (59); potentiel (57); câble (55); émetteur (55); émission (55); arc (53); service (52); tarif (50); sortie (49); caractère (47); phase (47); position (47); sensibilite (46); programme (45); cathode (44); entrée (44); réglage (44); bobine (43); connecteur (42); régulation (42); soudage (42); rendement (41); code (40); terre (39); couplage (38); polarisation (38); commutateur (37); connexion (37); diffusion (37); écran (37); capacité (36); support (36).

\begin{tabular}{lc}
\hline Fréquences & N. de formes diff. \\
\hline+500 & 3 \\
$499-200$ & 5 \\
$199-150$ & 4 \\
$149-100$ & 13 \\
$99-80$ & 9 \\
$79-60$ & 16 \\
$59-50$ & 18 \\
$49-50$ & 32 \\
$39-30$ & 46 \\
$29-25$ & 38 \\
$24-20$ & 72 \\
$19-15$ & 104 \\
$14-10$ & 158 \\
9 & 67 \\
8 & 69 \\
7 & 87 \\
6 & 143 \\
5 & 153 \\
4 & 224 \\
3 & 342 \\
2 & 745 \\
1 & 273 \\
\hline
\end{tabular}

Voici la liste des mots qui ne sont que constituants de termes: temps (186); système (171); dispositif (108); appareil (107); caractéristique (102); effet (102); coefficient (78); essai (70); bande 
(68); constante (59); courbe (56); fréquence (53); ionisation (53); chambre (50); détecteur (50); rapport (47); angle (46); distorsion (46); boîte (45); densité (44); poste (44); source (44); composante (42); lecture (42); retour (41); ligne (39); section (39); surface (38); espace (37); état (37); taux (37); pouvoir (36).

Pour atteindre $50 \%$ des occurrences, il faut ajouter à ces 100 mots les 223 mots suivants dans la liste de fréquence, ce qui veut dire que $50 \%$ des occurrences sont produites par $0,07 \%$ des mots $\mathrm{du}$ vocabulaire. Les mots qui n'apparaissent qu'une fois correspondent à 49,3\% des mots différents.

\section{Termes et constituants: redondance et combinatoire}

La similarité des phénomènes de saturation de vocabulaire pour les textes continus et les nomenclatures tient, sur le plan formel, à des raisons différentes. Si la concordance des chiffres, en ce qui concerne la répétition des mots dans ces deux types de textes, existe, la nature des répétitions et celle des mots répétés n'est pas la même. Sur le plan de la communication, cette similarité est liée aux mécanismes de redondance inhérents au fonctionnement de tout code; du point de vue terminologique, la redondance est un procédé économique.

Dans l'étude de cette nomenclature, il faut considérer la redondance lexicale comme une combinatoire et une distinction doit être faite entre la réutilisation de mots et la réutilisation de termes. Dans le deuxième cas, il n'y a pas seulement répétition de monotermes, mais répétition de multitermes. Pour illustrer ce phénomène, nous ne disposons pas de statistiques sur l'ensemble des termes, mais sur 6867 termes, soit $67 \%$ du corpus, ce qui constitue une proportion suffisante pour tirer des conclusions valables.

Sur 6867 termes, 1040 termes, soit $15 \%$, servent à former d'autres termes, et parmi ceux-ci près de la moitié (504) sont des syntagmes. Les deux types de syntagmes engendrant d'autres termes sont, comme on pouvait s'y attendre, [N + Adj] et [N + Prép $\mathrm{N}$ ], les deux modèles syntagmatiques les plus fréquents. Des monotermes servent donc à former des multitermes qui à leur tour peuvent également en former d'autres. La redondance lexicale est due en majeur partie à la présence de séries dans la nomenclature.

\section{Une étude de cas: les séries avec courant}

On distingue deux types de séries: les séries de syntagmes à tête identique et celles formées par les déterminations communes à plusieurs termes. Certains termes font partie des deux catégories 
de série. Nous ne possédons pas de statistiques permettant d'établir quelle est la proportion de termes réutilisés comme têtes de syntagmes puisque les chiffres énumérés précédemment concernent aussi bien les termes déterminés que les termes déterminants. Pour étudier cette question nous allons examiner la répartition des occurrences de courant, lequel arrive en tête des termes réutilisés.

Il existe 3C4 occurrences de courant dont 167 comme tête de syntagme. Cette série à tête identique est formée de 632 mots dont 178 formes différentes pour un taux de saturation N/V de $27 \%$. Ce pourcentage peut être considéré élevé, si l'on ne tenait compte que du nombre de termes, puisqu'il correspond au taux de saturation observé dans une tranche de 2000 termes de cette même liste.

Le modèle $[\mathrm{N}+$ Prép + (Dét) $+\mathrm{N}]$ engendre 64 termes, soit plus du tiers de la série, et il connaît des expansions en série comme dans les exemples suivants:

1. courant de court-circuit

2. courant de court-circuit permanent

3.a. courant de court-circuit monophasé permanent

3.b. courant de court-circuit biphasé permanent

3.c. courant de court-circuit triphasé permanent

Par l'addition de déterminations au terme courant, nous obtenons les termes 1, 2 et 3 qui sont, les uns par rapport aux autres, en relation subordonnée. Les termes 3.a. 3.b. 3.c. sont en relation coordonnée.

Le modèle $[\mathrm{N}+\mathrm{AJ}]$ engendre 35 termes et connaît également des expansions de série:

1. courant présumé

2. courant présumé établi

3. courant présumé coupé

4. courant présumé symétrique

Dans l'analyse des termes 2, 3 et 4, sans l'existence du terme 1 , il est facile d'appliquer la même analyse que dans: un individu présumé innocent, comme en langue générale c'est-à-dire: [courant [présumé établi]]. Or, en tenant compte du système de relations paradigmatiques propres à la terminologie, il faut plutôt privilégier l'analyse: [courant [présumé] [établi]] ce qui peut apparaître choquant au premier abord. Reprenons l'analyse avec le terme 4: courant présumé symétrique. Le courant n'est pas présumé ... symétrique, il est + [présumé] et + [symétrique]. L'hypothèse porte bel et bien sur l'existence d'un courant et non sur une de ses caractéristiques. Il existe peut-être un courant et, si ce courant existe, il est soit [coupé], soit [établi], soit [symétrique], etc. Quant 
au «sens» de ces syntagmes, il faut avouer qu'il demeure le même quelle que soit l'analyse choisie, ce qui est parfaitement explicable puisque le meilleur argument que l'on puisse invoquer pour étayer la thèse de la coordination des déterminants est celui de l'existence des termes courant coupé et courant établi. En fait, l'analyse en constituants du terme courant présumé coupé doit se faire comme suit:

premier niveau: [/courant présumé] coupé] deuxième niveau: [courant présumé] [courant coupé].

Ce type d'analyse peut s'appeler analyse en constituants déterminés par pertinence terminologique. L'utilisation de deux termes pour en former un troisième valide l'hypothèse de la coordination des déterminants et justifie qu'on prenne en considération l'axe paradigmatique dans l'étude de la syntagmatique des terminologies.

On peut observer qu'il y a également combinaison de déterminations différentes, soit les déterminations [adj] et [prép $+\mathrm{N}$ ] comme dans les exemples suivants:

1. courant minimal de commande

2. courant minimal de maintien

3. courant critique de grille

4. courant inverse d'électrode

5. courant élémentaire de conduction

Dans les trois premiers termes, il y a insertion d'un adjectif (pour 1 et 2 c'est le même adjectif) dans des termes déjà existants: courant de commande, courant de maintien, courant de grille, ce qui donne une idée du degré relatif de figement des syntagmes. Pour ce qui est du terme 4, on ajoute une détermination [PR $+N$ ] au terme courant inverse. Le dernier cas est une fusion de deux termes: courant élémentaire et courant de conduction. Tous ces termes doivent être considérés comme constituants des termes donnés en exemple.

Si l'ensemble des termes de la série courant ne peut pas être représenté sous forme arborescente, ces termes sont cependant tous reliés à la même notion mère: «courant». La série ayant comme tête courant s'explique d'un point de vue notionnel à la lumière de la théorie scientifique à partir de laquelle se sont développées les industries électriques et électrotechniques.

Si une théorie physique n'admet tien de semblable au courant électrique, un petit nombre de critères, qui peuvent varier considérablement d'un cas à l'autre, suffiront à identifier les courants, même s'il n'y a aucun ensemble de règles spécifiant 
les conditions nécessaires et suffisantes de son identification. (Kuhn, 1969, p. 267, note)

Si la théorie scientifique n'est pas à même de fournir les règles d'identification des notions mères, la formulation de règles sémantiques, comme l'utilisation d'un système de traits distinctifs ou de catégories sémantiques permettant de systématiser le processus de discrimination des termes, $s$ 'avérera d'autant plus difficile. On est ici confronté aux limites de la fonction classificatrice que l'on prête souvent aux nomenclatures techniques. Or, l'approche classificatrice, estimée possible dans le cas des nomenclatures techniques, est jugée difficile voire impossible par Alain Rey (1979) dans le cas des nomenclatures scientifiques. La série courant offre un bel exemple de la quasi-impossibilité de départager clairement ce qui appartient en propre au domaine technique et ce qui appartient au domaine scientifique.

Par ailleurs, les déterminations sérielles avec courant peuvent prendre différentes formes: [prép $+\mathrm{N}$ ] comme dans entrée de courant, prise de courant; $[\mathrm{N}+$ adj] comme dans convertisseur de courant alternatif, et convertisseur de courant continu. Le terme courant continu est utilisé comme détermination dans 30 syntagmes et courant alternatif est présent dans 19 syntagmes. Ils peuvent être liés au reste du syntagme par différentes prépositions: $\grave{a}, d e, e n$, par et pour. Il existe également d'autres formations qui n'entraînent pas nécessairement des séries: $[N+N]$ comme dans caractéristique temps-courant et [N + Adj + Adj] comme dans caractéristique du courant coupé limité où courant coupé est un terme ainsi que courant coupé limité. Tous les multitermes formés avec courant et utilisés comme déterminations n'entraînent pas nécessairement des déterminations sérielles.

Un syntagme dont la tête est courant ne reçoit sa signification que de l'existence d'autres termes de la série et les différents courants sont distingués les uns des autres sans que la théorie physique ne fournisse de réelle définition du courant électrique lui-même. Si l'on avait à nommer un nouveau courant il faudrait tenir compte de la série, puisque les séries forment des paradigmes. En effet, les termes entretiennent entre eux des relations paradigmatiques qui ne sont pas sans influer sur leur formation. De même, il existe deux types de relations paradigmatiques dans une nomenclature: la relation paradigmatique directe, celle qu'un terme entretient avec les termes de la série de syntagmes à tête identique à laquelle il appartient, et la relation paradigmatique oblique, celle qu'un terme entretient avec les termes d'une autre série lorsqu'une sous-expression significative de ce terme appartient à une autre série. Par exemple, le terme déclencheur a maximum de courant aura une relation paradigmatique directe avec les termes de la série 
déclencheur et une relation paradigmatique oblique avec les termes de la série courant.

De l'examen de ces données, il faut conclure qu'on ne peut étudier la syntagmatique des termes, les relations entre les constituants, sans tenir compte de l'existence de séties, c'est-à-dire sans tenir compte des relations paradigmatiques. La longueur d'un syntagme terminologique, par exemple, peut être conditionnée par le nombre et le type de relations paradigmatiques entretenues avec

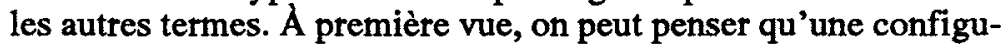
ration de 14 éléments (dont cinq substantifs) n'apparaissant qu'une seule fois dans le corpus $(0,00019 \%$ des termes) devrait être considérée comme une sorte de résidu sans réelle importance dans l'étude de la formation des termes. Or, sur les 296 configurations de substantifs, $52 \%$ n'apparaissent qu'une seule fois; il semble alors difficile de les mettre de côté sans tenter de les expliquer. Si de tels termes semblent être des phénomènes d'exception lorsqu'ils sont analysés sur le plan syntagmatique, leur caractère singulier s'estompe la plupart du temps sous l'angle paradigmatique. Ces termes existent parce qu'ils sont formés de termes et parce qu'ils s'inscrivent dans des séries.

La combinabilité des termes peut également servir à expliquer les différences de figement que l'on observe généralement entre les composés de la langue générale et ceux des langues de spécialité. Un terme désigne bien une seule notion, mais, puisque certains de ses constituants peuvent être des termes qui, combinés à d'autres termes, sont susceptibles d'en engendrer d'autres, le lien entre de tels constituants est plus lâche ou différent, comme l'a montré l'exemple de courant présumé coupé. Cette combinabilité fait partie du potentiel de dénomination d'une nomenclature.

\section{Applications en terminotique}

On a vu que la dénomination, en tant que mécanisme linguistique, est une articulation des plans syntagmatique et paradigmatique. Ce mécanisme produit des termes ayant les caractéristiques suivantes: organisation en séries, construction à partir de matrices et répétitions d'éléments de vocabulaire. L'exposition de telles caractéristiques révèle le potentiel de dénomination d'une nomenclature.

Le potentiel de dénomination constitue un élément de définition important. Dans un dictionnaire informatisé, pour chaque terme, il devrait être possible, sur demande, d'obtenir la liste des termes avec lesquels il se trouve en relation paradigmatique directe et celle des termes avec lesquels il entretient des relations paradigmatiques obliques. En effet, en envisageant d'un point de vue pratique les statistiques présentées, on peut affirmer que, si dans 
un domaine technoscientifique, on devait nommer une nouvelle notion, il y a de fortes probabilités pour que le nouveau terme soit un syntagme et qu'au moins un des constituants soit présent dans le vocabulaire de la nomenclature du domaine. Par conséquent, advenant le cas, plutôt fréquent, de l'absence de l'équivalent recherché dans la consultation d'un glossaire, la connaissance de la série dans laquelle le terme recherché doit s'insérer fournit par elle-même des éléments de réponse.

Sur le plan technique, la connaissance des matrices terminogéniques permet de procéder automatiquement et de manière économique à la reconnaissance des relations paradigmatiques, même dans les cas où un terme servant de constituant à un autre terme n'y est pas présent linéairement.

De plus, l'adoption d'un standard, relativement à la forme des entrées d'un dictionnaire spécialisé, permettrait l'échange et la mise en commun de travaux terminologiques au plan international. En effet, moins de $10 \%$ des publications technoscientifiques se font en français et cette situation implique d'énormes besoins de traduction. Pour répondre à une telle demande, les ouvrages terminographiques multilingues constituent des outils précieux que l'on doit multiplier et perfectionner si l'on veut suivre le courant des développements de la science et de la technique sans avoir à le faire dans une langue étrangère.

On ne peut opposer le concept de langue générale à celui de langues de spécialité qu'à partir de la dichotomie expérience partagée par l'ensemble des locuteurs/expérience partagée par des sous-ensembles de locuteurs. Un système de termes, allié à un système de définitions, révèle l'expérience propre à une sphère d'activité. Chaque définition terminologique doit tenter de cerner un "concept maximum» atteint au niveau supérieur d'une échelle allant du [- spécialisé] au [+ spécialisé], le degré de spécialisation pouvant être déterminé en fonction du sous-ensemble de locuteurs visé. De plus, il ne saurait être question de tout définir, car chaque sphère d'activité possède des concepts considérés comme des $a$ priori par ses spécialistes.

Dans un système de termes, du point de vue conceptuel, chaque terme doit être défini par rapport à l'ensemble dont il fait partie; du point de vue formel, ces rapports s'expriment par la présence de deux types de séries: les séries de syntagmes à tête identique et les séries de syntagmes à détermination commune. L'analyse syntagmatique d'un multiterme ne peut souvent être effectuée sans connaître les relations paradigmatiques que ce terme entretient avec les autres termes. Il existe donc une articulation des plans paradigmatique et syntagmatique et cette charnière constitue une structure linguistique propre à la dénomination. Cette 
structure linguistique engendre une dynamique interne propre à une nomenclature et cette dynamique, nous la nommons «potentiel de dénomination». Nous proposons de l'utiliser dans l'édification d'un système de définitions informatisé.

Cette étude de la dénomination, en tant que mécanisme linguistique, et de ses relations avec l'organisation conceptuelle n'est encore qu'embryonnaire, mais déjà ses applications sont plus que prometteuses. Pour cette éternelle question des mots et des choses, on voit actuellement se manifester un nouvel intérêt fouetté par les exigences de l'intelligence artificielle. C'est pourquoi il y a fort à parier que nous verrons dans les années à venir se développer ce champ d'investigation, à moins qu'il ne se révèle être une boîte de Pandore!

Université McMaster 


\section{Références}

Auger, P. (1978). «La Syntagmatique terminologique, typologie des syntagmes et limite des modèles en structure complexe». Table ronde sur les problèmes de découpage du terme, AILA, Office de la langue française du Québec.

Baudot, J. (1988). «Une minibanque de terminologie multilingue». Terminogramme, 46.

Boulanger, J.-C. (1988). «Le Syntagme terminologique: un projet de recherche». Terminogramme, 46.

Dubuc, R. (1978). «Découpage de l'unité terminologique». Table ronde sur les problèmes de découpage du terme, AILA, Office de la langue française du Québec.

Eco, U. (1986). Semiotics and the Philosophy of Language. Indiana University Press.

GuilBert, L. (1965). La Formation du vocabulaire de l'aviation. Paris, Larousse.

(1967). «La Dérivation syntagmatique dans les vocabulaires scientifiques et techniques". Les Langues de spécialité, Actes du stage de St-Cloud, AIDELA.

(1975). La Créativité lexicale. Paris, Larousse.

Haman, J. (1980). «Dictionaries and Encyclopedias». Lingua, 50.

JACKENDOFP, R. (1983). Semantics and Cognition. MIT Press.

Kant, E. (éd. 1976). Critique de la raison pure. Paris, Flammarion.

KuHN, T.S. (éd. 1983). La Structure des révolutions scientifiques, avec postface de 1969. Paris, Flammation.

LiCHNERowicz, A. (1987). «Universalité des mathématiques et compréhension du réel» in les Scientifiques parlent..., sous la direction d'Albert Jacquard. Paris, Hachette.

Merćux, I. (1984). Dictionnaire explicatif et combinatoire du français contemporain. Montréal, les Presses de l'Université de Montréal, «Recherches lexico-sémantiques I».

(1988). Dictionnaire explicatif et combinatoire du français contemporain. Montréal, les Presses de l'Université de Montréal, «Recherches lexico-sémantiques II».

Muller, Ch. (1968). Initiation à la statistique linguistique. Paris, Larousse.

Polanyi, M. (1958). Personal Knowledge. Towards a post-critical Philosophy. The University of Chicago Press.

(1946). Introduction (1964) Science Faith and Society. The Univetsity of Chicago Press. 
POPPER, C. (1985) «Truth and Approximation to Truth» (1960) in Popper Selections, ed. by David Miller. Princeton University Press.

Portelance, C. (1986). «A propos du rôle des matrices terminogéniques...» Terminogramme, 40.

(1987). «Fertilisation terminologique ou insémination terminologique artificielle». Meta, 32(3).

(1989). «Syntagmes et parádigmes». Meta, 34(3).

(à paraître, 1991). «Fondement linguistique de la terminologie». Meta, 36(1).

Rey, A. (1979). La Terminologie: noms et notions. Paris, PUF, "Que sais-je?», 1780.

(1977). Le Lexique: image et modeles du dictionnaire d̀ la lexicologie. Paris, Armand Colin.

WierzBickA, A. (1985). Lexicography and conceptual analysis. Karoma Publishers inc. 